\title{
3 Research Square

\section{Inhibition of PAR1 attenuates meningeal macrophage activation and vascular inflammation in a rat model of thrombin-induced hydrocephalus}

\section{Xiaodi Hao}

Zhejiang University https://orcid.org/0000-0003-0584-9217

Xianghua Ye

Zhejiang University

Dan Shen

Zhejiang University

Jiawen Li

Zhejiang University

Chensheng Le

Zhejiang University

Lu-sha Tong ( $\nabla 2310040 @ z j u . e d u . c n)$

Zhejiang University

Feng Gao ( 2202012@zju.edu.cn )

Zhejiang University

\section{Research}

Keywords: hydrocephalus, meningeal macrophage, inflammation, thrombin, PAR1 antagonist

Posted Date: December 19th, 2019

DOl: https://doi.org/10.21203/rs.2.19125/v1

License: (c) (1) This work is licensed under a Creative Commons Attribution 4.0 International License.

Read Full License 


\section{Abstract}

\section{Background}

Our previous studies demonstrated that intraventricular injection of thrombin could induce hydrocephalus. The inflammation of subarachnoid space plays a key role in hydrocephalus. As thrombin, inducing coagulation, could contribute to inflammation, its effects on subarachnoid space have not been well studied. Macrophagic dysfunction may contribute to this course. However, the mechanisms that how thrombin affects macrophage in subarachnoid space have not been illustrated. Our aim was to explore the possible role that macrophage played in thrombin-induced meningeal inflammation, and to furtherly understand its contribution during thrombin-induced hydrocephalus.

\section{Methods}

There were two parts in this study. Firstly, rats had an intraventricular injection of saline or thrombin. Secondly, rats received thrombin injection with vehicle or PAR1 antagonist treatment.

Immunofluorescence staining was applied to observe the activation of meningeal macrophage and the expression of NeuN in the cortex. Meanwhile, the expression of intercellular adhesion molecule 1 (ICAM1) in meningeal vessels were tested to detect the vascular inflammation. Western blot was applied to measure the secretion of pro-inflammatory cytokines (IL-1 $\beta$ and IFNY).

\section{Results}

Our results demonstrated that intraventricular injection of thrombin caused significant activation of meningeal macrophages, vascular inflammation, and neuron loss. Inhibition of PAR1 pathway attenuated the M1 polarization of meningeal macrophage, reduced the inflammatory infiltrations and prevented the neuron loss, as well as hydrocephalus after thrombin injection.

\section{Conclusions}

Clinically available PAR1 antagonists may offer a novel therapeutic approach candidate for the prevention or the management of inflammation in hemorrhage-induced hydrocephalus.

\section{Background}

Hydrocephalus is an independent risk factor of hemorrhage-associated morbidity [1]. Our previous study found that thrombin played a critical role in hydrocephalus [2,3]. Cerebrospinal fluid (CSF) is mainly produced in choroid plexus and absorbed in subarachnoid space, and the key point of hydrocephalus should be the changes in formation or absorption of CSF [4-6]. Thrombin is produced immediately after hemorrhage stroke and plays an essential role in brain damage after intracerebral hemorrhage (ICH) $[2,3$, 7, 8]. Thrombin could disrupt blood-cerebrospinal fluid (CSF) barrier and generate communicating hydrocephalus via proteinase-activated receptor 1 (PAR1) pathway, which leads to vascular endothelialcadherin (VE-cadherin) downregulation in choroid plexus $[2,3,9]$. 
Inflammation in the ventricular system is strongly associated with the blood-CSF barrier and hydrocephalus formation [9-14]. Subarachnoid space plays a critical role in cerebrospinal fluid absorption, the dysfunction of which would contribute to hydrocephalus $[5,12,15]$. Acute inflammatory cell infiltration in subarachnoid space caused the transformation of pial cells into macrophages, promoted fibroblasts and extracellular matrix deposition leading to subarachnoid fibrosis and hydrocephalus $[12,15,16]$. The meningeal macrophage is one type of non-parenchymal macrophages that mediate immune responses at brain boundaries $[17,18]$. The relationship between thrombin and meningeal inflammation is still unknown. In this study, the thrombin-induced inflammation in subarachnoid space was investigated.

The high-affinity thrombin receptor, PAR1, has been implicated in mediating the interaction between coagulation and inflammation [19]. The role of inflammation and PAR1 in thrombin-induced hydrocephalus needs to be further elucidated. In this study, we aimed to explore the role of thrombin in brain injury and further illustrate the mechanism of hemorrhage-associated hydrocephalus.

\section{Methods}

\section{Animals model}

Animal protocols were approved by and conducted in accordance with the ethical guidelines of the Zhejiang University Animal Experimentation Committee and were in complete compliance with the National Institutes of Health Guide for the Care and Use of Laboratory Animals. A total of 32 male Sprague-Dawley rats (3-month old, Zhejiang University Laboratories), at the weight of 280-320 g, were used in this study. Animals were anesthetized with pentobarbital (50 mg/kg intraperitoneally (IP)) and were positioned in a stereotaxic frame. A cranial burr hole $(1 \mathrm{~mm})$ was drilled and a 26-gauge needle was inserted stereotaxically into the right lateral ventricle (coordinates: $0.6 \mathrm{~mm}$ posterior, $4.5 \mathrm{~mm}$ ventral, and $1.6 \mathrm{~mm}$ lateral to the bregma). Saline or thrombin ( $3 \mathrm{U}$ in $50 \mu \mathrm{l})$ was infused using a microinfusion pump (World Precision Instruments). The needle was removed after injection, the burr hole was filled with bone wax, and the skin incision was sutured.

\section{Experimental groups}

In brief, the rats were randomly divided into two parts. First, rats received an intracerebroventricular (ICV) injection of 3 units rat thrombin (Sigma-Aldrich, St Louis, MO, USA) in saline or saline alone (50 $\mu$ l) in 7 minutes and were euthanized for intracardiac perfusion and the brains were used for Western blot $(n=4$ for each group), brain histology ( $\mathrm{n}=4$ for each group) at 24 hours. Second, rats were treated with vehicle (1\% dimethyl sulfoxide in saline) or PAR1 inhibitor (SCH79797, Abcam, USA, 25 $\mu \mathrm{g} / \mathrm{kg}$ ) immediately while thrombin infusion. Rats were treated with $\mathrm{SCH} 79797$ at a dosage accordingly $[3,8]$. The rats were euthanized at 24 hours after injection and the brains were used for western blot ( $n=4$ for each group) and brain histology ( $\mathrm{n}=4$ for each group).

\section{Immunohistochemistry and HE staining}


Rats were anesthetized with pentobarbital $(100 \mathrm{mg} / \mathrm{kg}, \mathrm{IP})$ and perfused with $4 \%$ paraformaldehyde in $0.1 \mathrm{~mol} / \mathrm{L}$ phosphate-buffered saline $(\mathrm{pH} 7.4)$. The brains were removed and kept in $4 \%$ paraformaldehyde for 24 hours and then immersed in $30 \%$ sucrose for 2 to 3 days at $4{ }^{\circ} \mathrm{C}$. Brains were embedded in optimal cutting temperature compound and 18- $\mu \mathrm{m}$ thick slices cut using a cryostat (LEICA, CM3050S). Immunohistochemistry and HE staining were performed as previously described. The primary antibodies were goat anti-lba1 (1:400 dilution; Abcam, Cambridge, MA, USA), mouse anti-CD68 (1:100 dilution; Abcam, Cambridge, MA, USA), mouse anti-NeuN (1:200 dilution; Abcam, Cambridge, MA, USA), rabbit antiCD206 (1:200 dilution; Abcam, Cambridge, MA, USA).

\section{Western blot analysis}

Briefly, brain tissue was immersed in western sample buffer and lysed. The lysate was centrifuged at $12000 \mathrm{r} / \mathrm{min}$ for $30 \mathrm{~min}$ at $4^{\circ} \mathrm{C}$ and the supernatant was collected. Protein concentration was determined by the BCA protein assay kit (Pierce, Thermo Scientific, USA). Protein samples were separated using SDSpolyacrylamide gel electrophoresis and transferred to the nitrocellulose membrane. After blocking with $5 \%$ nonfat milk, the membranes were incubated with primary antibodies anti- IL-1 $\beta$ (1:2000 dilution; Abcam, Cambridge, MA, USA), anti- IFNy (1:2000 dilution; Abcam, Cambridge, MA, USA) and anti-GAPDH (1:3000; KangChen, Shanghai, China) at $4^{\circ} \mathrm{C}$ overnight. After repeated washes, the membranes were reacted with antibodies against rabbit IgG (1:3000; Multi Sciences, China) or mouse IgG (1:3000; Multi Sciences, China) for $2 \mathrm{~h}$ at room temperature. The relative densities of bands were analyzed with ImageJ software.

\section{Statistical analysis}

Values are given as means \pm SD. Student t-tests were used to compare the data between two groups. Differences were considered significant at $p<0.05$.

\section{Results}

Thrombin promoted the activation of meningeal macrophage

We first observed the accumulation of inflammatory cells in subarachnoid space. Our findings suggested that inflammation is widespread throughout the subarachnoid space after intraventricular injection of thrombin (Fig. 1a). In this study, Iba1 was used to label meningeal macrophages, CD68 was used as a marker of M1 macrophages and CD206 was used as a marker of M2 phenotype. CD68 or CD206 positive inflammatory cells were counted and the results were expressed as positive cells per millimeter of meninges. In areas of subarachnoid space, there was abundant CD68 positive meningeal macrophages in the thrombin group compared with markedly lower numbers in the saline group (115.4 \pm 28.41 versus $37.7 \pm 17.19$ positive cells $/ \mathrm{mm}$ in the saline group, $p<0.01$, Fig. $1 \mathrm{~b}$ ). It indicated that thrombin induced the M1 polarization of meningeal macrophage in subarachnoid space. While there was a potential increase tendency in the distribution of CD206 positive macrophages between thrombin and saline group (122.4 \pm 60.76 versus $63.57 \pm 14.72$ positive cells $/ \mathrm{mm}$ in the saline group, $p=0.1088$, Fig. $1 \mathrm{c}$ ). These results 
demonstrated that thrombin remarkably promoted M1 polarized meningeal macrophage rather than $\mathrm{M} 2$ phenotypic macrophage.

\section{$\underline{\text { Thrombin induced vascular inflammation in subarachnoid space }}$}

The adherence and migration of leukocytes through the endothelium of blood vessels in subarachnoid space is an important event which triggers the early inflammatory response [20]. In subarachnoid space, prominent ICAM1 staining of endothelial cells in the thrombin group was observed (Fig. 2a). A significant rise in the expression of ICAM1 in endothelial cells and meningeal macrophages after thrombin injection was observed. At 24 hours after thrombin injection, the higher levels of proinflammatory cytokines (IL-1 $\beta$, IFNY) were also detected compared to the saline group (Fig. 2b, c).

\section{Thrombin caused hydrocephalus and neuron loss}

Ventricular volumes were measured by brain sections at 10 levels ( $50 \mu \mathrm{m}$ in thickness) and NeuN and Iba1 immunostaining were performed in primary somatosensory cortex. The results showed that thrombin injection caused significant hydrocephalus $\left(22.28 \pm 6.062\right.$ versus $8.46 \pm 1.95 \mathrm{~mm}^{3}$ in saline group, $\mathrm{p}<0.01$, Fig. 3a) and a significant reduction in numbers of neurons at 24 hours after intraventricular thrombin injections compared with the saline group (Fig. 3b).

\section{Inhibition of PAR1 alleviated the meningeal macrophage activation in subarachnoid space}

To examine the role of PAR1 in thrombin-induced inflammation, $\mathrm{SCH} 79797$ was used. $\mathrm{SCH} 79797$ is a specific PAR1 antagonist which resulted in less inflammation infiltration in subarachnoid space (Fig. 4a). Additionally, fewer CD68 positive cells were observed in the SCH79797 group compared to the vehicle group ( $61.39 \pm 16.43$ versus $126 \pm 36.6$ positive cells/mm in the vehicle group, $p<0.05$, Fig. $4 \mathrm{~b})$. And there were less CD206 positive cells in subarachnoid space compared to vehicle group $(77.59 \pm 29.74$ versus $134.9 \pm 46.07$ positive cells $/ \mathrm{mm}$ in vehicle group, $p=0.0815$, Fig. $4 \mathrm{c}$ ).

SCH79797 reduced the infiltration and adhesion of inflammatory cells in subarachnoid space

Furthermore, thrombin-induced vascular inflammation was also suppressed by treatment with SCH79797. Inhibition of PAR1 attenuated the expression of ICAM1 (Fig. 5a) and proinflammatory cytokines (IL-1 $\beta$, IFNY) (Fig. 5b, c).

\section{SCH79797 suppressed the thrombin-induced hydrocephalus and neuron loss}

Treatment with $\mathrm{SCH} 79797$ protected the neuron loss and produced a negative effect on ventricle enlargement (11.45 \pm 2.254 versus $20.79 \pm 3.297 \mathrm{~mm}^{3}$ in the vehicle group, $p<0.01$, Fig. 6).

The meningeal macrophage activation was associated with the neuron loss and hydrocephalus

To better evaluate the association between meningeal inflammation and thrombin-induced hydrocephalus, a detailed quantitative analysis of ventricular volume and CD68 positive cell numbers 
was carried out in all cases with and without the thrombin injection. The density of CD68+ meningeal macrophages reflected the severity of hydrocephalus and neuron loss in the early phase of thrombininduced brain injury (Fig. 7).

\section{Discussions}

The major findings of this study are as follows: (1) thrombin promoted M1 polarization of meningeal macrophage; (2) thrombin induced vascular inflammation in subarachnoid space; (3) inhibition of PAR1 attenuated the inflammatory response in subarachnoid space after thrombin injection; (4) meningeal inflammation was associated with severity of thrombin-induced hydrocephalus and neuron loss.

Thrombin is an important component of the coagulation cascade and plays an essential role in hydrocephalus $[2,3,7]$. Lateral ventricular volumes in rats were significantly larger in 24 hours rather than in the following day 1 to 28 after intraventricular hemorrhage [2]. Our previous study found that thrombin disrupted the blood-CSF barrier via VE-cadherin downregulation and led to communicating hydrocephalus $[2,3,9]$. And the inflammation response was previously proved to be closely associated with the formation of hydrocephalus $[11,12,21,22]$. Systemic inflammation is a therapeutic target in acute ischemic stroke [23,24]. Also, it was indicated that anti-inflammation treatment could alleviate spontaneous hydrocephalus [25]. In the current study, the role of inflammation in thrombin-induced hydrocephalus was investigated.

Non-parenchymal brain macrophages are mononuclear phagocytes that are increasingly recognized to be critical players in the diseases of the central nervous system [25-28]. There are three types of central nervous system macrophages between parenchyma and circulation- namely, meningeal, perivascular and choroid plexus macrophages $[17,18,28]$. Meningeal inflammation has been identified as a key feature of hydrocephalus $[12,15,21]$ and may contribute to the extensive cortical pathology that accompanies progressive disease [29-32]. Meningeal cells are involved in cortical development, fibrotic scar formation, brain inflammation, and neurodegenerative disorders such as Multiple Sclerosis (MS) and Alzheimer's disease (AD) and other brain disorders [31-33]. Here, our study aimed to explore whether meningeal macrophages were involved in the inflammation of subarachnoid space and the formation of hydrocephalus $[12,15]$. Two macrophage populations were found in the central nervous system, corresponding to the proinflammatory M1 and "alternatively activated" anti-inflammatory M2 classes [18, 27]. The M1 macrophages are characterized by the high expressions of oxygen intermediates and proinflammatory cytokines which are closely associated with brain injury [25, 34]. While the M2 phenotype is considered to be involved in the promotion of tissue remodeling [18, 34]. In our study, a significant increase in M1 polarization was observed in meningeal macrophages and many of them are CD68 positive after thrombin injection. Interestingly, there was no significant difference in M2 macrophages which were CD206 positive between saline and thrombin group.

One of the most important features of proinflammatory macrophage is the secretion of pro-inflammatory cytokines [34]. In this study, activated endothelial cells and inflammatory infiltrations were observed after 
thrombin injection, pro-inflammatory cytokines were also significantly increased. Cytokines (like IL-1 $\beta$, IFNY) were shown to decrease VE-cadherin protein levels and induce endothelial barrier dysfunction [35, 36]. Intercellular adhesion molecule 1 (ICAM1), induced by IL-1 $\beta$, IFNY [37], is best known for its role in leukocyte adhesion and typically expressed on endothelial cells and cells of the immune system [20,38]. Additionally, ICAM1 could mediate Src activation [39, 40], which is involved in the regulation of VEcadherin $[3,8]$. In this study, the increased expression of ICAM1, IL-1 $\beta$, and IFNY after thrombin injection were observed, which highlighted the role of the inflammatory response in thrombin-induced hydrocephalus. Furthermore, a growing body of evidence demonstrated that leukocyte-endothelial adhesion interaction could lead to posthemorrhagic vasospasm which also could exacerbate the neuron loss [41-43].

To investigate the relationship between inflammation and thrombin-induced brain injury, the ventricular volume and the neuron loss in the cortex were analyzed. Interestingly, the level of infiltrating CD68 positive macrophages in meninges, correlated modestly with the severity of hydrocephalus and neuron loss. Rats with increased meningeal inflammation had a more severe brain injury. These results suggested that diffuse meningeal inflammation may contribute to the pathological mechanisms driving the progression of hydrocephalus and cortical neuronal pathology. These findings are consistent with other observations in meningeal inflammation [29-32].

The cellular signaling effects of thrombin are mediated by protease-activated receptors (PARs) $[2,3,44]$. PAR1, PAR3, and PAR4 can be activated by thrombin, PAR2 is activated by coagulation factors VIla and $\mathrm{Xa}$, but not by thrombin [44]. While lesser extent PAR3 and PAR4 are regulated in reactive brain injury [45], PAR1 plays a more extensive role in thrombin signaling $[8,19,46]$. The activation of PAR1 disrupts vascular integrity $[3,8]$, results in neurotoxicity $[47,48]$ and increases secondary brain injury after stroke[2, $7,46]$. In our study, inhibition of PAR1 reduced the meningeal inflammation, neuron loss, and hydrocephalus. It is possible that anti-inflammation will provide novel therapeutic targets for hydrocephalus management in the future.

\section{Conclusion}

These results demonstrated that meningeal macrophages were activated by intraventricular thrombin injection and it also exaggerated neuron loss and peri-vascular inflammation in the subarachnoid space. The clinically available PAR1 antagonists could reverse the meningeal injury and inflammation, thus might offer a new therapeutic approach for management in ventricular hemorrhage-induced hydrocephalus.

\section{Abbreviations}

CNS: Central nervous system

CSF: Cerebrospinal fluid 
ICAM1: intercellular adhesion molecule 1

$\mathrm{ICH}$ : intracerebral hemorrhage

VE-cadherin: vascular endothelial cadherin

PAR1: proteinase-activated receptor 1

\section{Declarations}

\section{Ethics approval and consent to participate}

Human volunteers did not participate in the study. Animal experiments were conducted after the approval from the Zhejiang University Animal Experimentation Committee.

\section{Consent for publication}

Not applicable.

\section{Availability of data and materials}

The datasets used and/ or analyzed during the current study are available from the corresponding author on reasonable request.

\section{Competing interests}

The authors declare that they have no competing interests.

\section{Funding}

This work was supported by the National Natural Science Foundation of China (81471168, F. Gao) and National Natural Science Foundation of China (NSFC) (81500991) to L.S. Tong.

\section{Authors' contributions}

$\mathrm{XH}, \mathrm{XY}, \mathrm{DS}, \mathrm{JL}$, and $\mathrm{CL}$ performed the experiments, analyzed the data, prepared the figures and wrote the manuscript. LT and FG designed the experiments, interpreted the data, and edited the manuscript.

\section{Acknowledgements}

Not applicable.

\section{References}

1. Nieuwkamp, D.J., et al., Treatment and outcome of severe intraventricular extension in patients with subarachnoid or intracerebral hemorrhage: a systematic review of the literature. Journal of 
Neurology, 2000. 247(2): p. 117-121.

2. Gao, F., et al., Hydrocephalus after Intraventricular Hemorrhage: The Role of Thrombin. Journal of Cerebral Blood Flow \& Metabolism, 2013. 34(3): p. 489-494.

3. Hao, X.D., et al., Thrombin disrupts vascular endothelial-cadherin and leads to hydrocephalus via protease-activated receptors-1 pathway. CNS Neuroscience \& Therapeutics, 2019.

4. Bering, E.A. and O. Sato, Hydrocephalus: changes in formation and absorption of cerebrospinal fluid within the cerebral ventricles. Journal of neurosurgery, 1963. 20(12): p. 1050-1063.

5. Orešković, D. and M. Klarica, Development of hydrocephalus and classical hypothesis of cerebrospinal fluid hydrodynamics: facts and illusions. Progress in neurobiology, 2011. 94(3): p. 238258.

6. Del Bigio, M.R. and D.L. Di Curzio, Nonsurgical therapy for hydrocephalus: a comprehensive and critical review. Fluids and Barriers of the CNS, 2016. 13(1): p. 3.

7. Klebe, D., et al., Dabigatran ameliorates post-haemorrhagic hydrocephalus development after germinal matrix haemorrhage in neonatal rat pups. Journal of Cerebral Blood Flow \& Metabolism, 2016. 37(9): p. 3135-3149.

8. Yan, J., et al., Role of SCH79797 in Maintaining Vascular Integrity in Rat Model of Subarachnoid Hemorrhage. Stroke, 2013. 44(5): p. 1410-1417.

9. Li, T., et al., Thrombin-induced TGF-beta 1 pathway: a cause of communicating hydrocephalus post subarachnoid hemorrhage. Int J Mol Med, 2013. 31(3): p. 660-6.

10. Goldim, M.P., et al., Oxidative stress in the choroid plexus contributes to blood-cerebrospinal fluid barrier disruption during sepsis development. Microvascular Research, 2019. 123: p. 19-24.

11. Karimy, J.K., et al., Inflammation-dependent cerebrospinal fluid hypersecretion by the choroid plexus epithelium in posthemorrhagic hydrocephalus. Nature Medicine, 2017. 23(8): p. 997-1003.

12. Botfield, H., et al., Decorin prevents the development of juvenile communicating hydrocephalus. Brain, 2013. 136(9): p. 2842-2858.

13. Hofmann, S., et al., The tumour necrosis factor-alpha induced vascular permeability is associated with a reduction of VE-cadherin expression. European journal of medical research, 2002. 7(4): p. 171176.

14. Xiang, J., et al., The choroid plexus as a site of damage in hemorrhagic and ischemic stroke and its role in responding to injury. Fluids and Barriers of the CNS, 2017. 14(1): p. 8.

15. Tan, Q., et al., Cannabinoid receptor 2 activation restricts fibrosis and alleviates hydrocephalus after intraventricular hemorrhage. Brain Research, 2017. 1654: p. 24-33.

16. Feng, Z., et al., Intraventricular administration of urokinase as a novel therapeutic approach for communicating hydrocephalus. Translational Research, 2017. 180: p. 77-90.e2.

17. Goldmann, T., et al., Origin, fate and dynamics of macrophages at central nervous system interfaces. Nature Immunology, 2016. 17: p. 797. 
18. Li, Q. and B.A. Barres, Microglia and macrophages in brain homeostasis and disease. Nature Reviews Immunology, 2018. 18(4): p. 225.

19. Samad, F. and W. Ruf, Inflammation, obesity, and thrombosis. Blood, 2013. 122(20): p. 3415-3422.

20. Kivisäkk, P., et al., Human cerebrospinal fluid central memory CD4+T cells: Evidence for trafficking through choroid plexus and meninges via P-selectin. Proceedings of the National Academy of Sciences, 2003. 100(14): p. 8389-8394.

21. Zhou, F., et al., Hydrocephalus Secondary to Chronic Meningitis. CNS Neuroscience \& Therapeutics, 2014. 20(3): p. 296-297.

22. Lattke, M., et al., Nuclear Factor KB Activation Impairs Ependymal Ciliogenesis and Links Neuroinflammation to Hydrocephalus Formation. The Journal of Neuroscience, 2012. 32(34): p. 11511.

23. Dziedzic, T., Systemic inflammation as a therapeutic target in acute ischemic stroke. Expert Review of Neurotherapeutics, 2015. 15(5): p. 523-531.

24. Chamorro, Á., et al., Neuroprotection in acute stroke: targeting excitotoxicity, oxidative and nitrosative stress, and inflammation. The Lancet Neurology, 2016. 15(8): p. 869-881.

25. Gu, C., et al., Effects of minocycline on epiplexus macrophage activation, choroid plexus injury and hydrocephalus development in spontaneous hypertensive rats. Journal of Cerebral Blood Flow \& Metabolism, 2019: p. 0271678X19836117.

26. Faraco, G., et al., Perivascular macrophages mediate the neurovascular and cognitive dysfunction associated with hypertension. The Journal of clinical investigation, 2016. 126(12): p. 4674-4689.

27. Shechter, R., et al., Recruitment of beneficial M2 macrophages to injured spinal cord is orchestrated by remote brain choroid plexus. Immunity, 2013. 38(3): p. 555-569.

28. Herz, J., et al., Myeloid Cells in the Central Nervous System. Immunity, 2017. 46(6): p. 943-956.

29. Magliozzi, R., et al., A Gradient of neuronal loss and meningeal inflammation in multiple sclerosis. Annals of Neurology, 2010. 68(4): p. 477-493.

30. Choi, S.R., et al., Meningeal inflammation plays a role in the pathology of primary progressive multiple sclerosis. Brain, 2012. 135(10): p. 2925-2937.

31. Howell, O.W., et al., Meningeal inflammation is widespread and linked to cortical pathology in multiple sclerosis. Brain, 2011. 134(9): p. 2755-2771.

32. Bevan, R.J., et al., Meningeal inflammation and cortical demyelination in acute multiple sclerosis. Annals of Neurology, 2018. 84(6): p. 829-842.

33. Dragunow, M., Meningeal and choroid plexus cells-Novel drug targets for CNS disorders. Brain research, 2013. 1501: p. 32-55.

34. Sica, A. and A. Mantovani, Macrophage plasticity and polarization: in vivo veritas. The Journal of Clinical Investigation, 2012. 122(3): p. 787-795.

35. Sonar, S.A., et al., IFN-y promotes transendothelial migration of CD4+ T cells across the blood-brain barrier. Immunology \& Cell Biology, 2017. 95(9): p. 843-853. 
36. Cromer, W.E., et al., The effects of inflammatory cytokines on lymphatic endothelial barrier function. Angiogenesis, 2014. 17(2): p. 395-406.

37. Mehmet Yasar, K., et al., Detection of soluble intercellular adhesion molecule-1 and vascular cell adhesion molecule-1 in both cerebrospinal fluid and serum of patients after aneurysmal subarachnoid hemorrhage. Journal of Neurosurgery, 2004. 101(6): p. 1030-1036.

38. Hubbard, A.K. and R. Rothlein, Intercellular adhesion molecule-1 (ICAM-1) expression and cell signaling cascades. Free Radical Biology and Medicine, 2000. 28(9): p. 1379-1386.

39. Yan, M., et al., Endothelial cell SHP-2 negatively regulates neutrophil adhesion and promotes transmigration by enhancing ICAM-1-VE-cadherin interaction. The FASEB Journal, 2017. 31(11): p. 4759-4769.

40. Liu, G., et al., ICAM-1-activated SrC and eNOS signaling increase endothelial cell surface PECAM-1 adhesivity and neutrophil transmigration. Blood, 2012. 120(9): p. 1942.

41. Sills, A.K., Jr., et al., Endothelial Cell Expression of Intercellular Adhesion Molecule 1 in Experimental Posthemorrhagic Vasospasm. Neurosurgery, 1997. 41(2): p. 453-461.

42. Gallia, G.L. and R.J. Tamargo, Leukocyte-endothelial cell interactions in chronic vasospasm after subarachnoid hemorrhage. Neurological Research, 2006. 28(7): p. 750-758.

43. Revuelta, M., et al., Characterization of Gene Expression in the Rat Brainstem After Neonatal Hypoxic-Ischemic Injury and Antioxidant Treatment. Molecular Neurobiology, 2017. 54(2): p. 11291143.

44. Coughlin, S.R., Thrombin signalling and protease-activated receptors. Nature, 2000. 407(6801): p. 258.

45. Pompili, E., et al., Trimethyltin-induced differential expression of PAR subtypes in reactive astrocytes of the rat hippocampus. Molecular Brain Research, 2004. 122(1): p. 93-98.

46. Manaenko, A., et al., PAR-1 antagonist SCH79797 ameliorates apoptosis following surgical brain injury through inhibition of ASK1-JNK in rats. Neurobiology of disease, 2013. 50: p. 13-20.

47. Lee, P.R., et al., Protease-activated receptor-1 activation by granzyme $B$ causes neurotoxicity that is augmented by interleukin-1 $\beta$. Journal of neuroinflammation, 2017. 14(1): p. 131.

48. Yoon, H., et al., Kallikrein 6 signals through PAR1 and PAR2 to promote neuron injury and exacerbate glutamate neurotoxicity. Journal of neurochemistry, 2013. 127(2): p. 283-298.

\section{Figures}


a

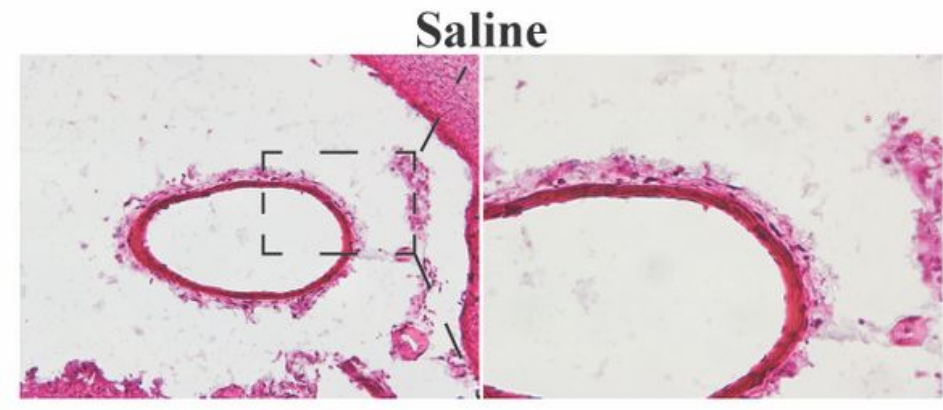

b

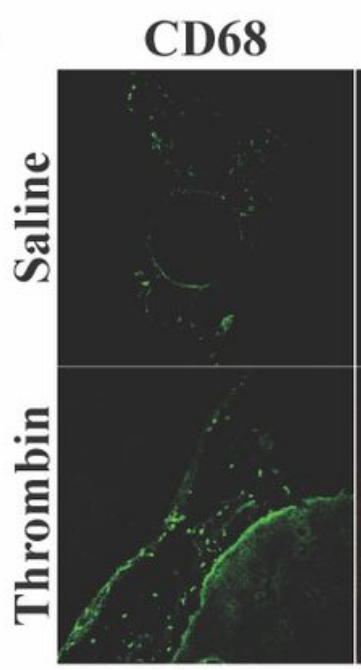

c

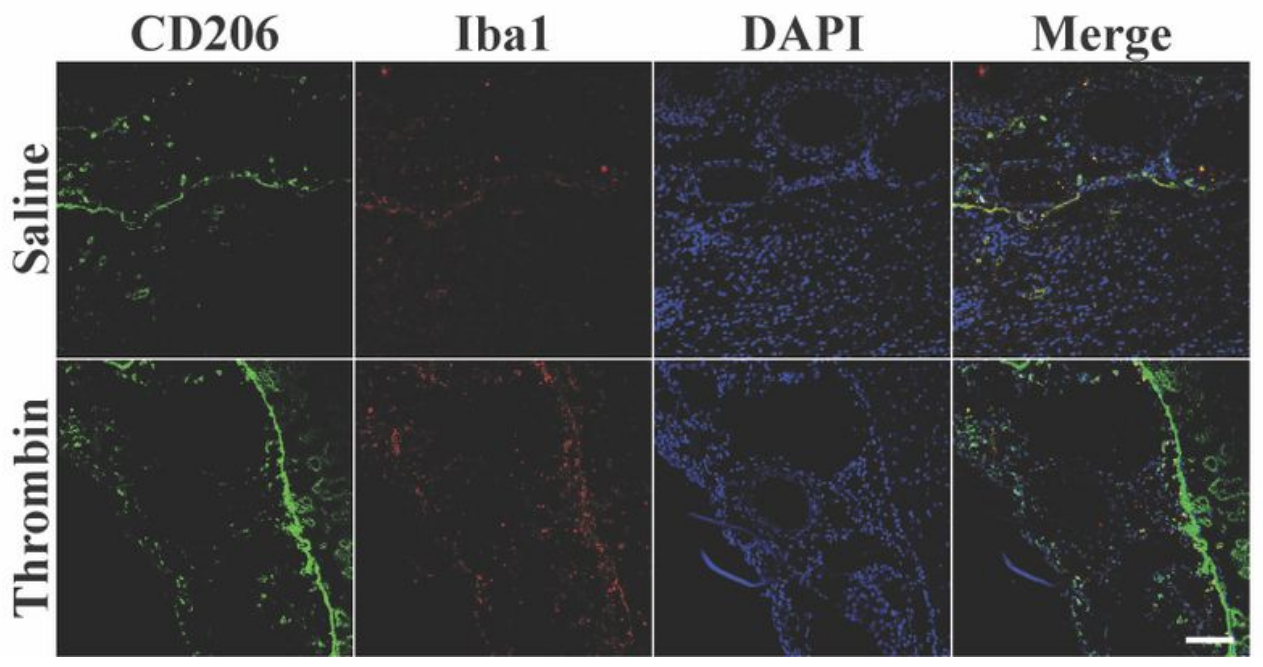

\section{Thrombin}

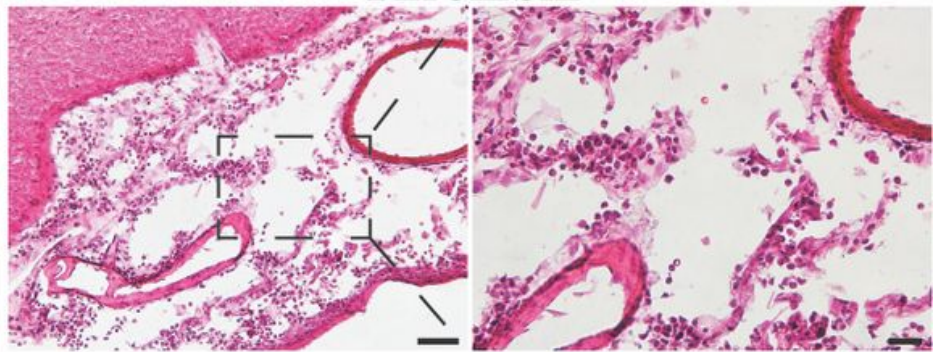

DAPI

Merge
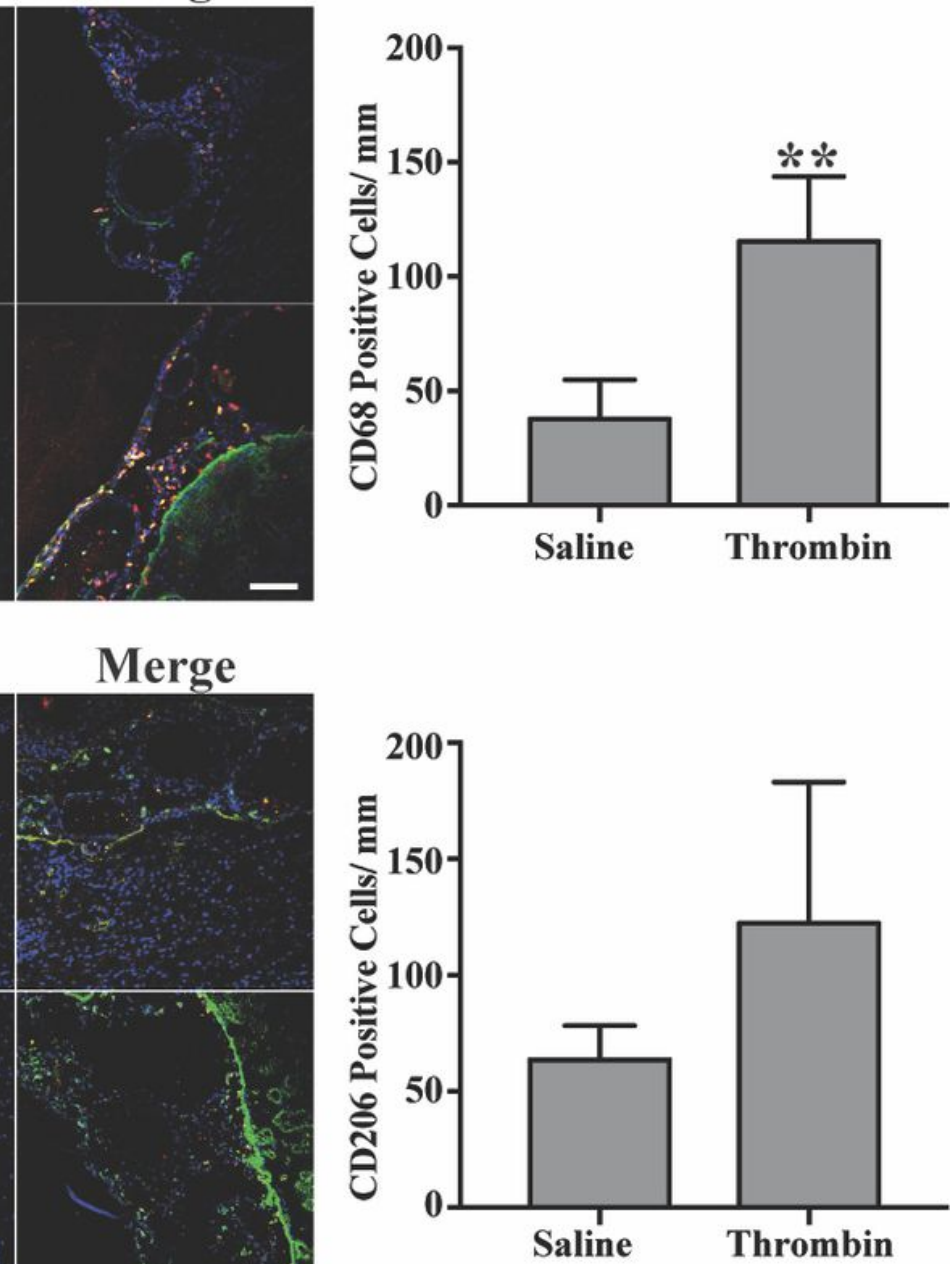

\section{Figure 1}

Distribution of inflammatory cells at 24 hours in subarachnoid space in the saline or thrombin group. (a) $\mathrm{H}$ \& E staining showed more inflammation infiltration in the thrombin group. Scale bar $=20 \mu \mathrm{m}$ (high magnification) and $50 \mu \mathrm{m}$ (low magnification). (b) CD68 and lba1 immunohistochemistry in rats injected with saline or thrombin. Scale bar $=200 \mu \mathrm{m}$. Values are means $\pm S D, n=4, * \star P<0.01$ vs saline group. (c) CD206 and Iba1 immunohistochemistry in saline or thrombin group. Scale bar=200 $\mu \mathrm{m}$. 
a

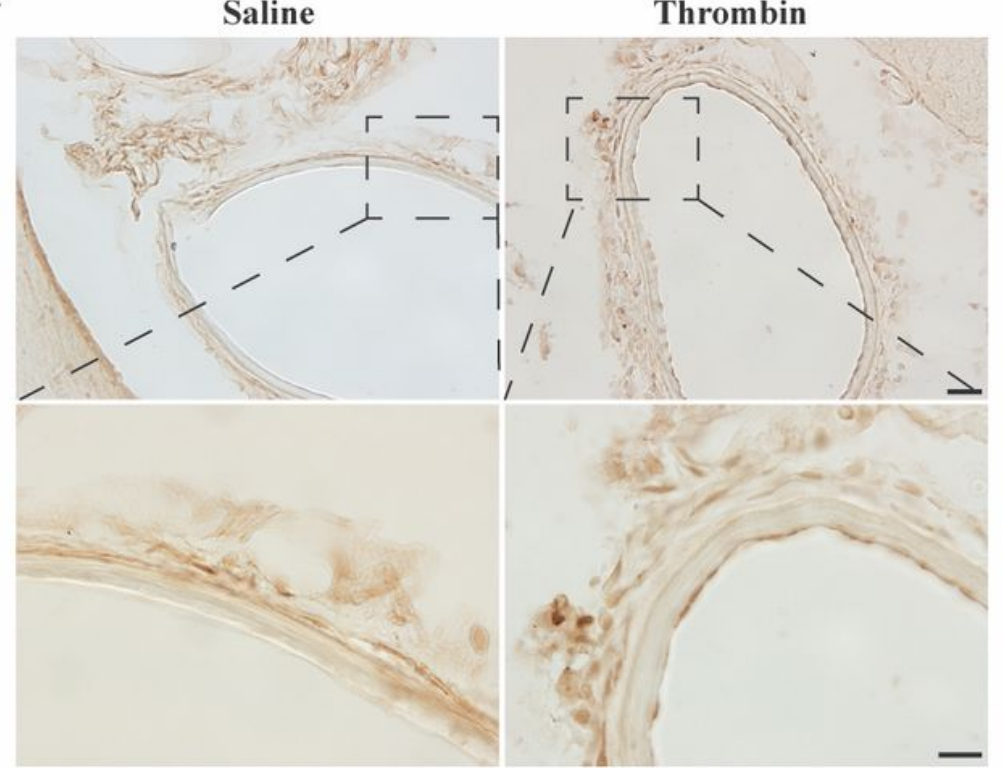

b

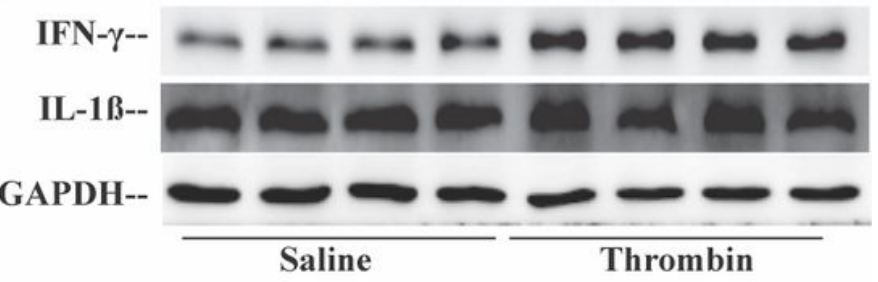

c
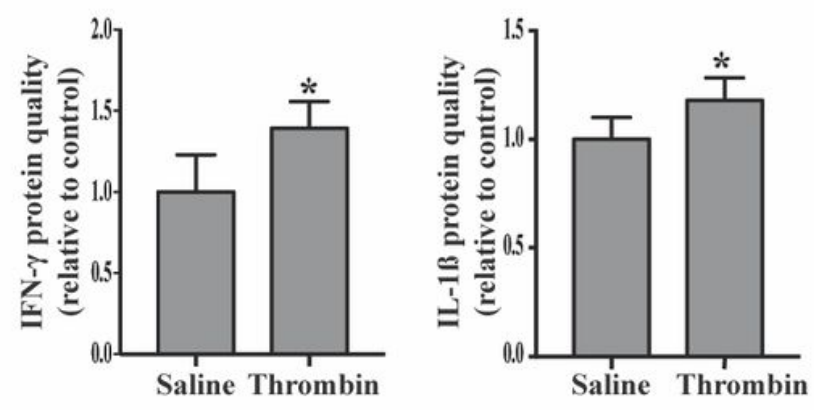

Figure 2

(a) Examples of ICAM1 staining at 24 hours after intracerebroventricular injection of saline or thrombin in subarachnoid space. Scale bar=10 $\mu \mathrm{m}$ (high magnification) and $20 \mu \mathrm{m}$ (low magnification). (b) The level of inflammatory cytokines was assessed by Western blot. (c) Quantitative analysis of inflammatory cytokines. Values are means $\pm S D, n=4$, * $P<0.05$ vs saline group

a

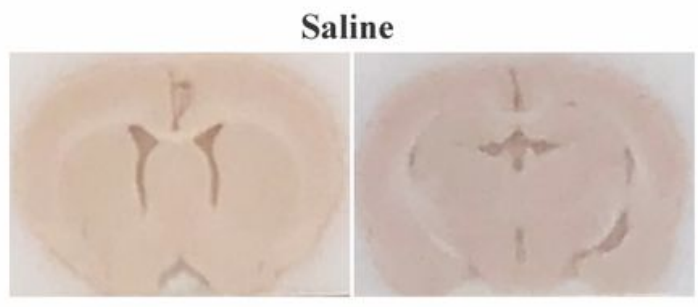

b

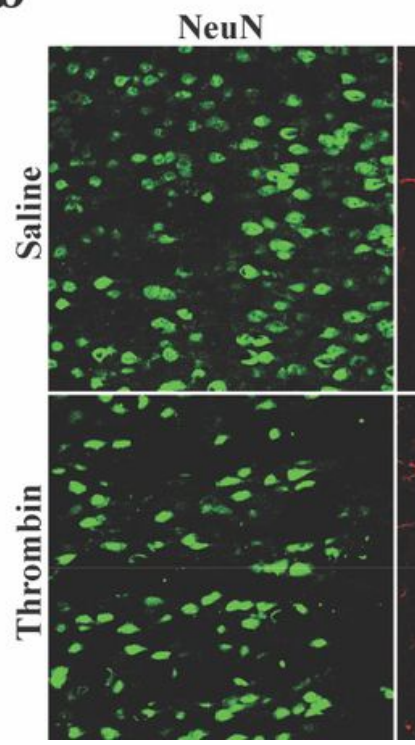

Iba1

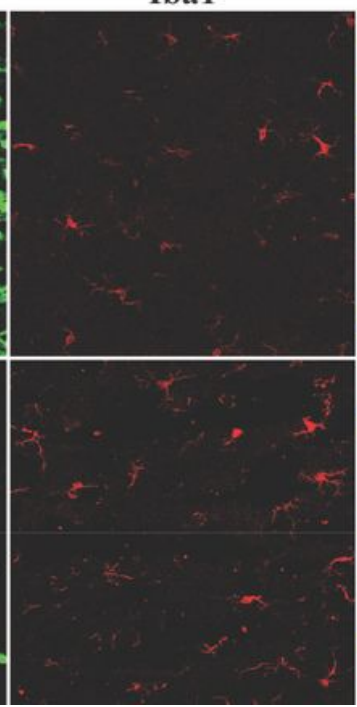

Thrombin

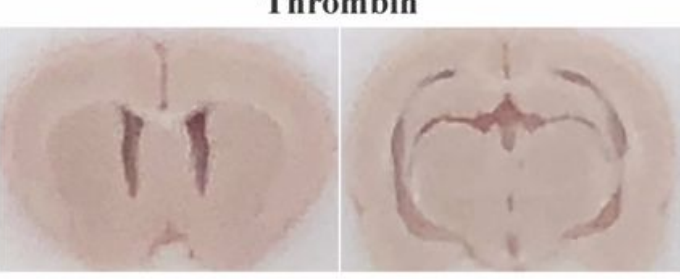

DAPI
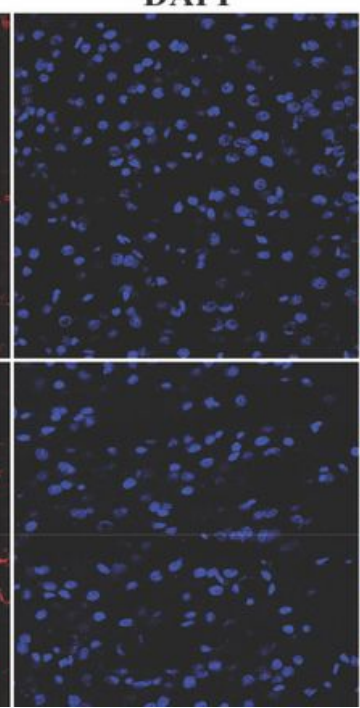

Merge
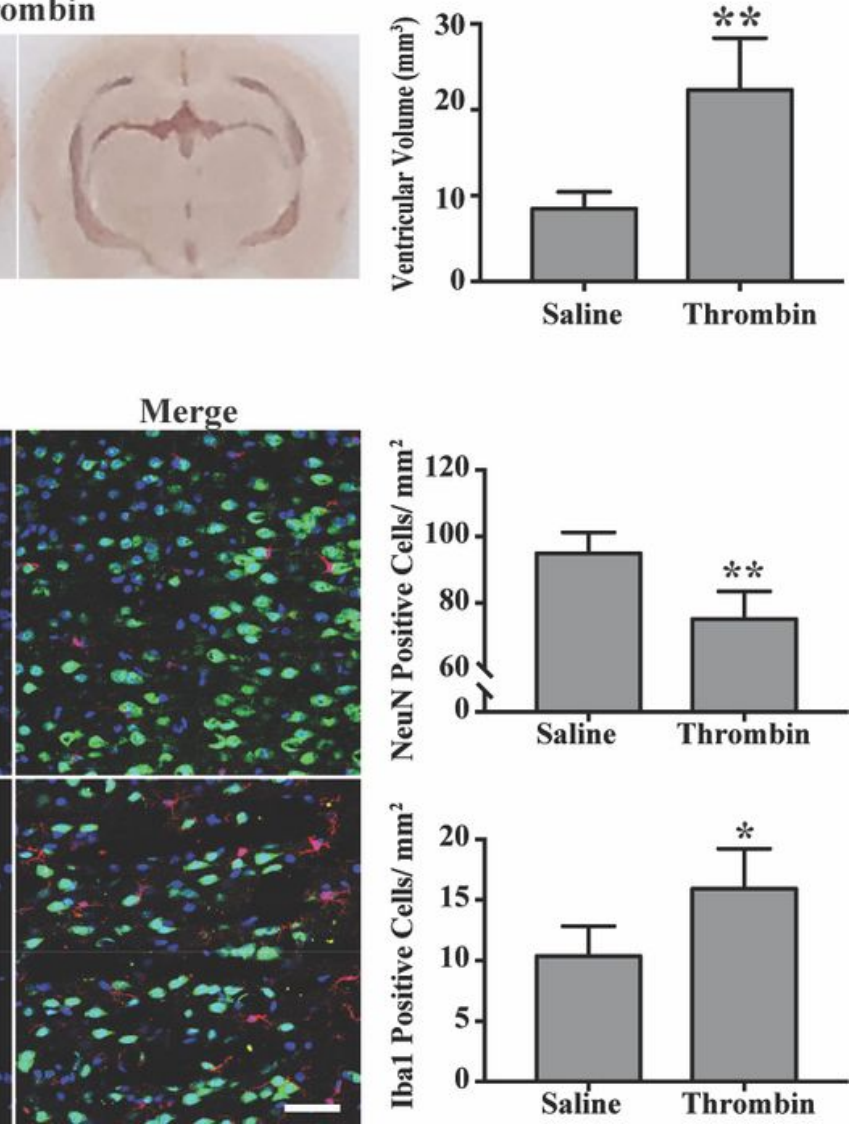


\section{Figure 3}

(a) Ventricles volumes were confirmed by examining coronal sections of frozen brains at 24 hours. $n=4$, $\star \star P<0.01$ vs saline group. (b) NeuN and Iba1 positive cells in the cortex. Scale bar $=50 \mu \mathrm{m}$. Values are means $\pm S D, n=4$, ${ }^{*}<0.05$ and ${ }^{\star *} P<0.01$ vs saline group.

a

Thrombin+vehicle

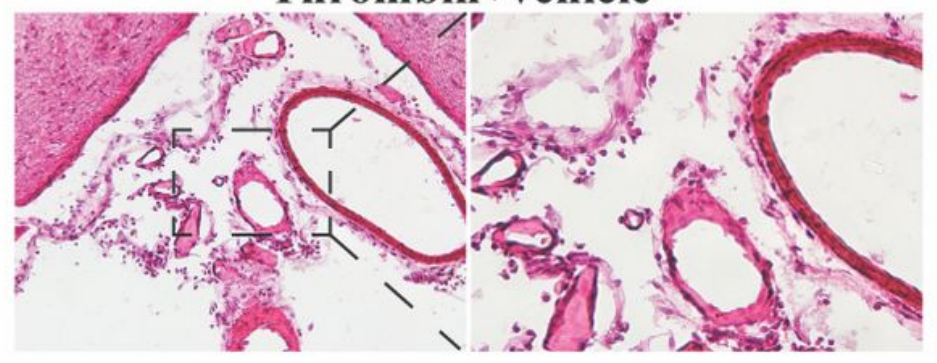

b

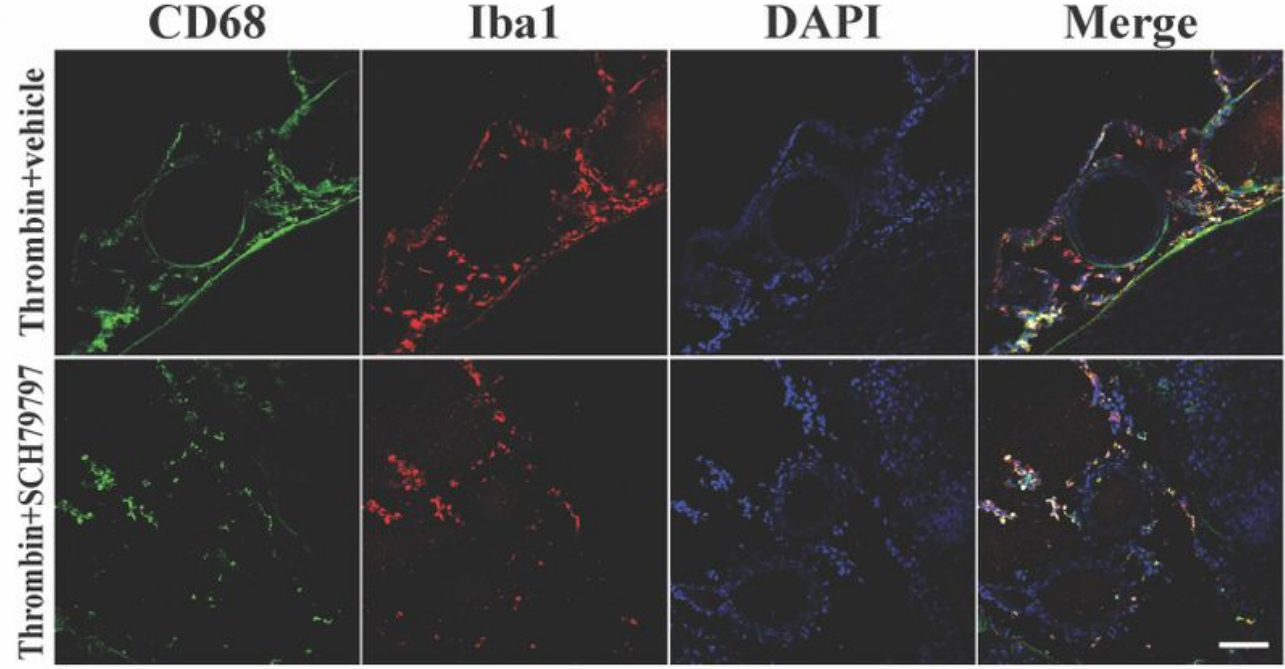

c

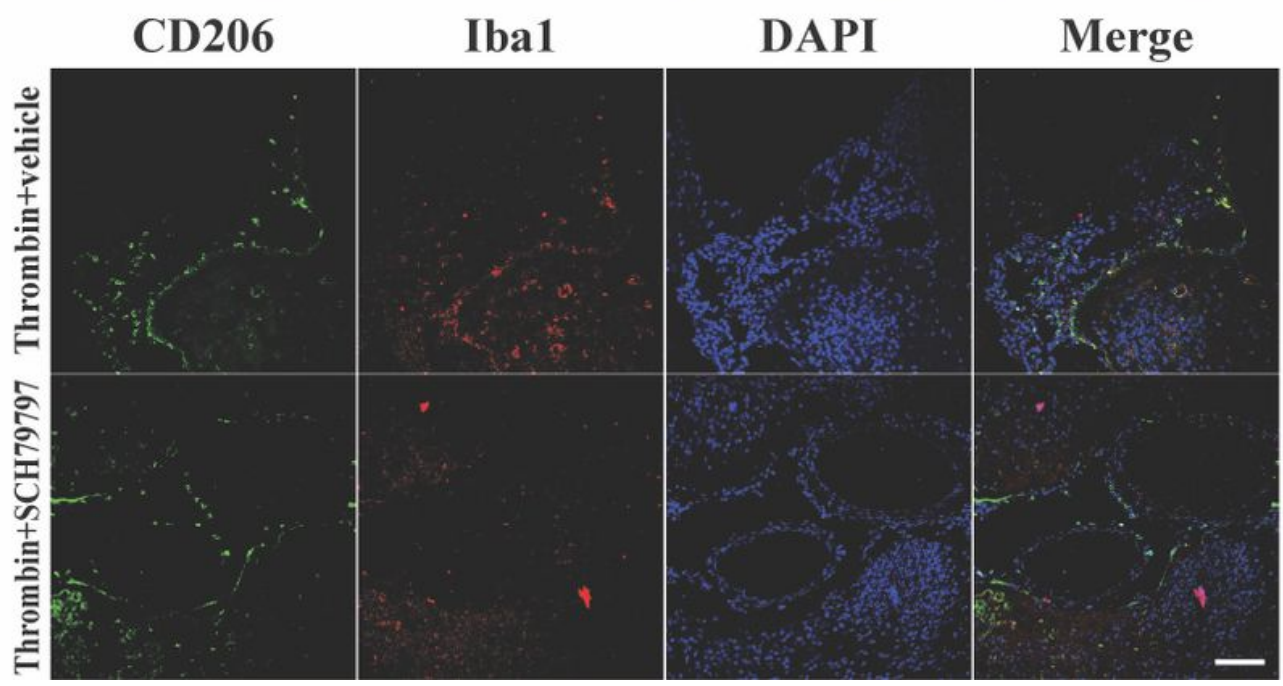

Thrombin+SCH79797
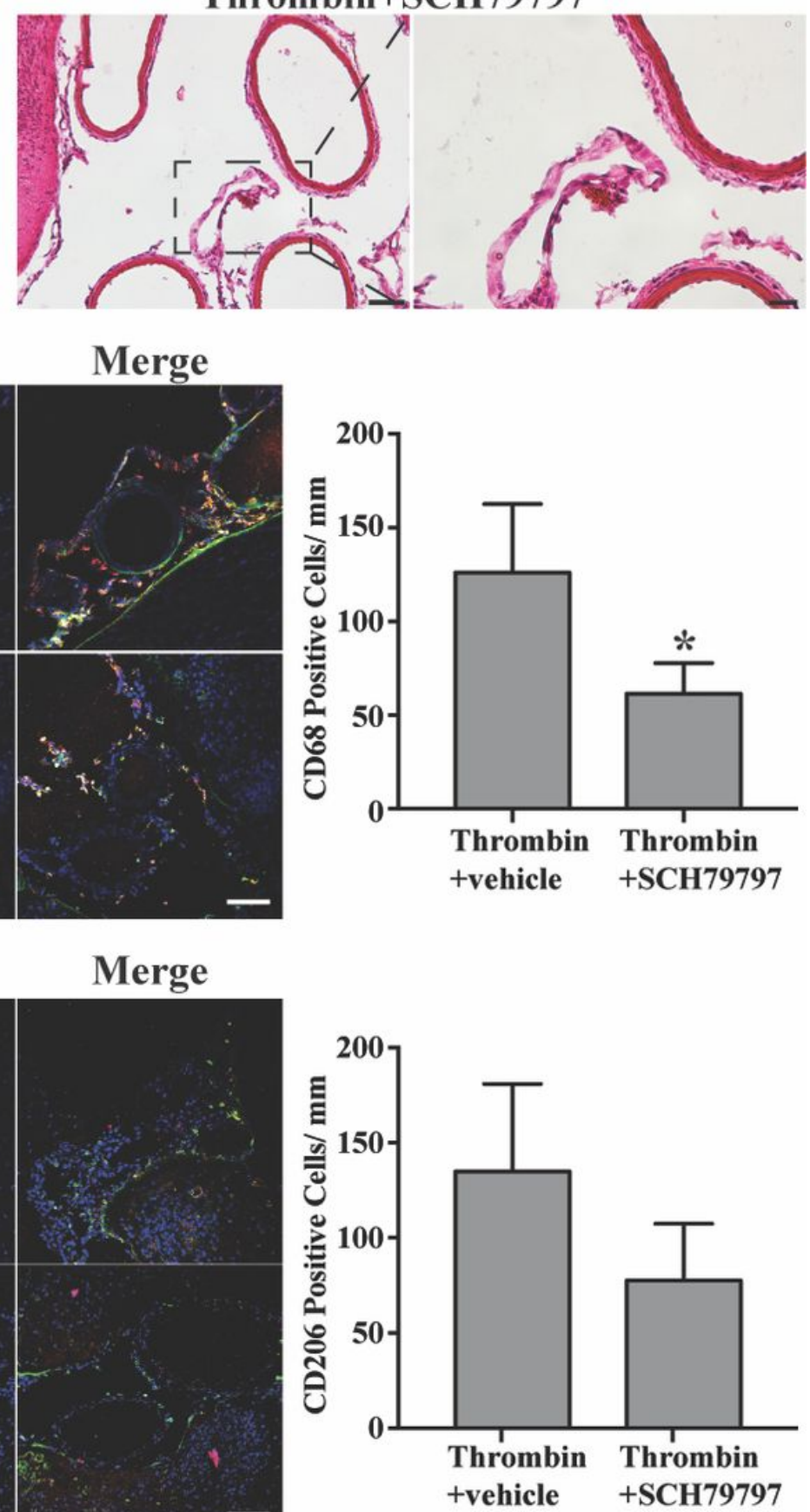

Figure 4

Distribution of inflammatory cells in the subarachnoid space in rats treated with vehicle or SCH79797. (a) $\mathrm{H} \& \mathrm{E}$ staining showed less inflammation infiltration in the $\mathrm{SCH} 79797$ group. Scale bar=20 $\mu \mathrm{m}$ (high magnification) and $50 \mu \mathrm{m}$ (low magnification). (b) CD68 and Iba1 positive cells in subarachnoid space. 
Scale bar $=200 \mu \mathrm{m}$. Values are means $\pm S D, n=4, * P<0.05$ vs vehicle group. (c) CD206 and lba1 immunohistochemistry in thrombin + vehicle or SCH79797 group. Scale bar=200 $\mu \mathrm{m}$. Values are means \pm $\mathrm{SD}, \mathrm{n}=4$.

a

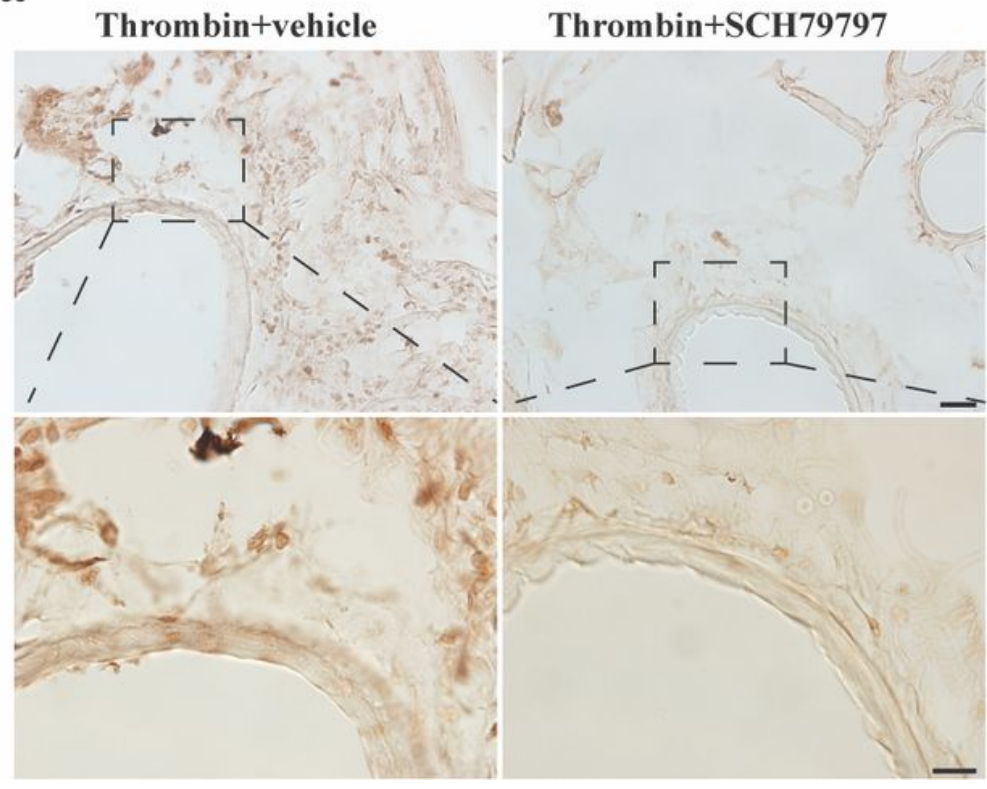

b

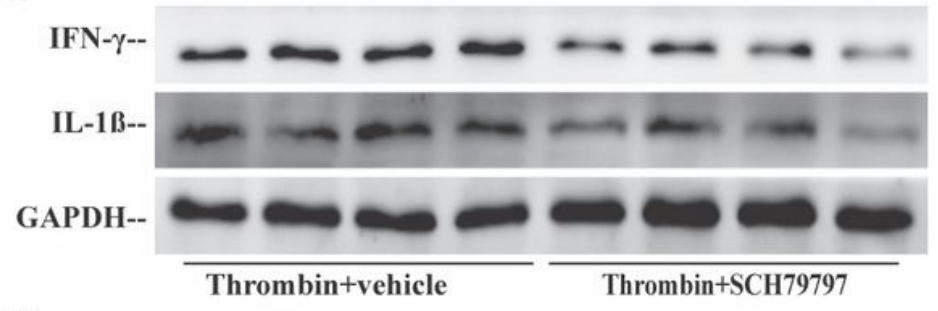

c

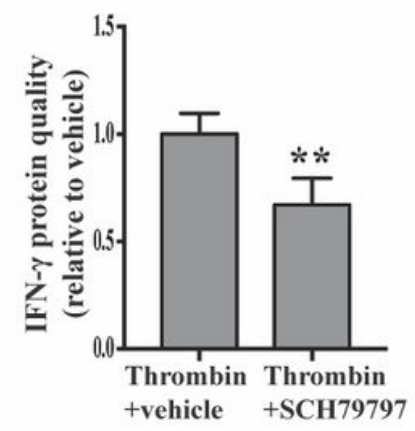

Figure 5

(a) ICAM1 staining in rats treated with vehicle or $\mathrm{SCH} 79797$. Scale bar=10 $\mu \mathrm{m}$ (high magnification) and $20 \mu \mathrm{m}$ (low magnification). (b) Inflammatory cytokines levels determined by Western blot and (c) quantitative analysis of inflammatory cytokines. Values are means $\pm S D, n=4,{ }^{*} P<0.05$ and ${ }^{*} P<0.01 v s$ vehicle group 
a

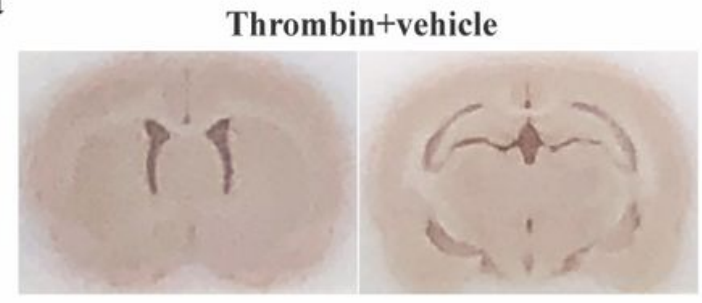

b

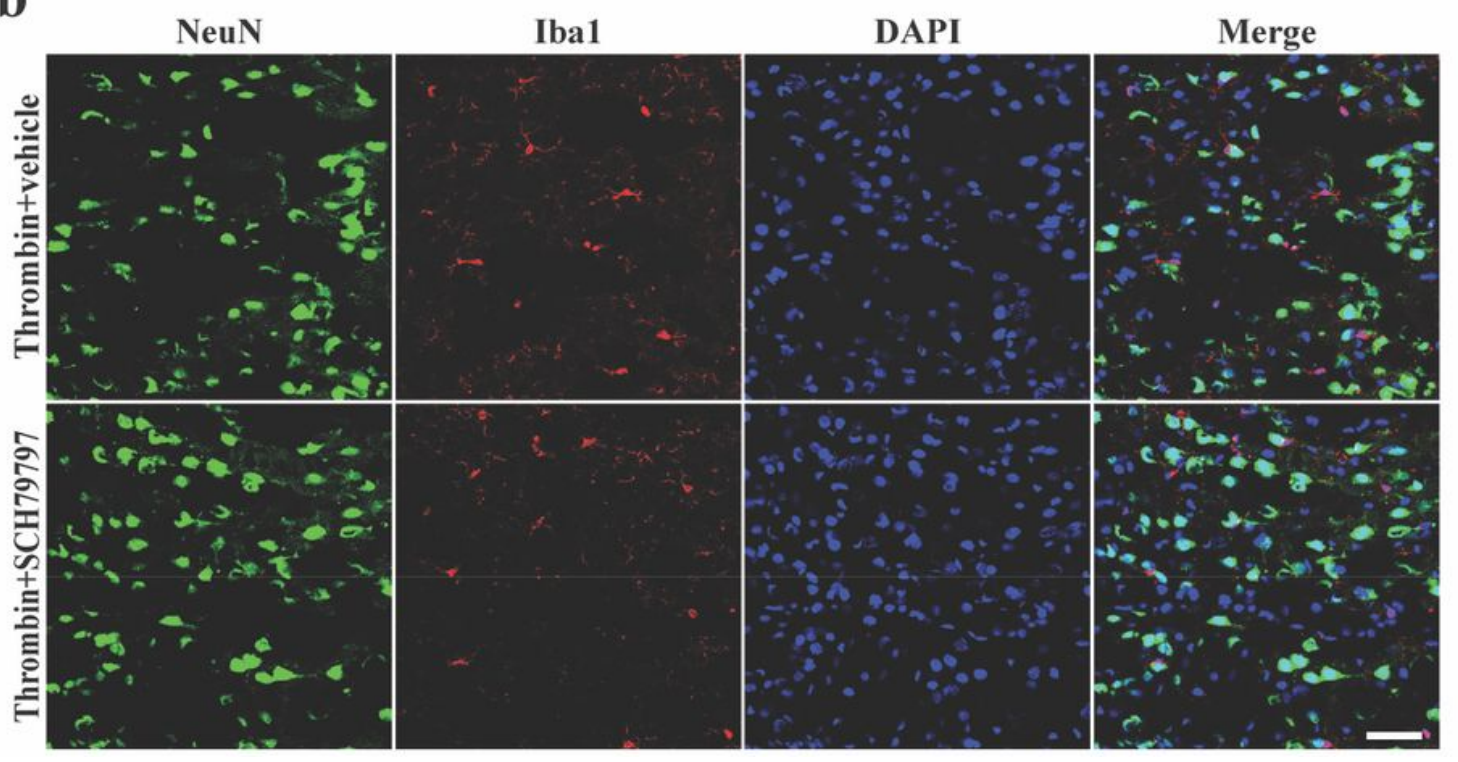

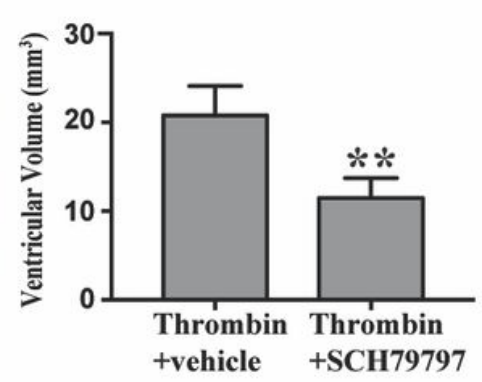

Figure 6

(a) Representative frozen sections from thrombin + vehicle or SCH79797 group. $n=4$, ${ }^{\star *} \mathrm{P}<0.01$ vs vehicle group. (b) Immunofluorescence staining of NeuN and Iba1 in the cortex. Scale bar $=50 \mu \mathrm{m}$. Values are means $\pm S D, n=4,{ }^{*} P<0.05$ and $* * P<0.01$ vs thrombin + vehicle group.

a

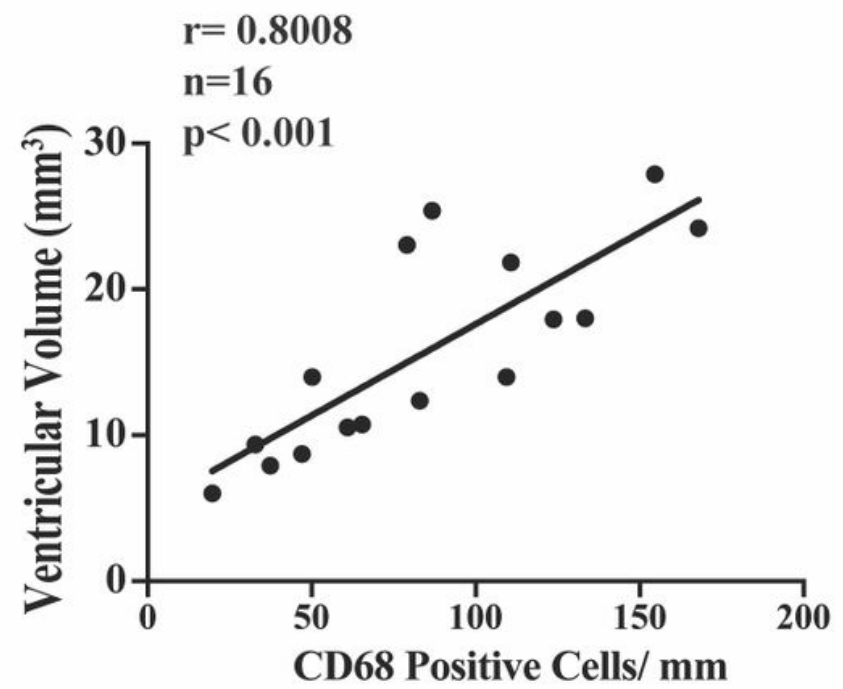

b

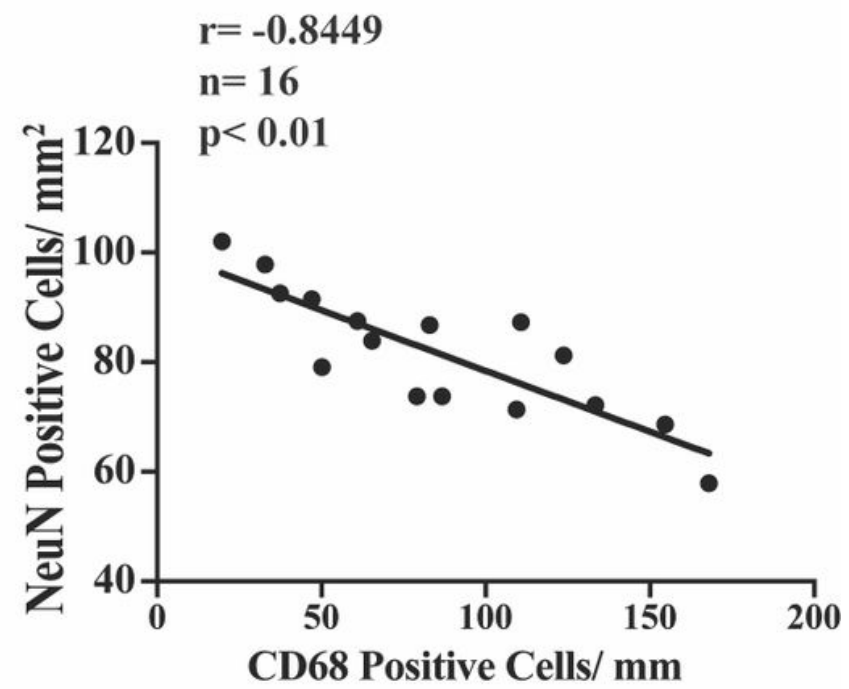

Figure 7 
Pearson's correlation analysis between CD68 positive cells and ventricular volume (a) or NeuN positive cells (b). 